Article

\title{
Numerical and Physical Experiments on Drag Torque in a Wet Clutch
}

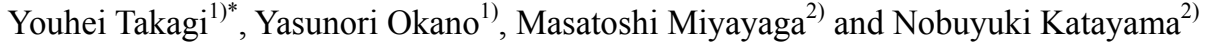 \\ ${ }^{1)}$ Department of Materials Engineering Science, Osaka University \\ 1-3 Machikaneyama, Toyonaka, Osaka 560-8531, Japan \\ ${ }^{2)}$ Research and Development Division, F.C.C. Co. Ltd. \\ 7000-46 Nakagawa, Hosoe, Kita-ku, Hamamatsu, Shizuoka 431-1304, Japan \\ *Corresponding author: takagi@cheng.es.osaka-u.ac.jp
}

( Manuscript received 14 May 2012; accepted 23 October 2012; published 15 November 2012 )

\begin{abstract}
The effect of flow field on drag torque in a wet clutch was examined through numerical and physical experiments. Three-dimensional hydrodynamic numerical simulations were carried out, and the drag torque was measured experimentally for a single wet clutch pack. Two-phase flow induced by aeration was visualized in the experiment. The wet clutch consists of two parallel circular plates. The plate with one side covered with the frictional material was rotated. The radial or circumferential grooves were made on the rotating disk by dividing the frictional material. The lubrication oil was supplied from the axial center, and ejected into the surrounding open boundary. At low rotation speeds, the oil flow was of single-phase, and the drag torque was linearly proportional to the rotation speed since the wall shear stress increased monotonically. In the single-flow regime, the slope of drag torque curve was controlled with the clearance between the clutch plates. The drag torque reached a peak value at a certain rotation speed, and it decreased gradually after the peak because of aeration. The peak of drag torque was controlled by both the flow rate of supplied oil and the arrangement of grooves on the frictional material. It also was found that the smooth ejection of oil and the enhancement of aeration led to the drag torque reduction.
\end{abstract}

Keywords: wet clutch, drag torque, aeration, visualization, numerical simulation

\section{Introduction}

In spite of significant progresses made in the development of alternative energy driven vehicles such as hybrid, fuelcell, and electric, it appears that the use of gasoline engine will still be dominant for the next few decades or so. Thus, the study of energy efficiency of gasoline engine vehicles is still important.

Energy losses in such vehicles are due to various components, and that of the powertrain is about $15 \%$ of the total amount. A clutch is one of the components of the powertrain assembly. In its engagement state, the clutch transmits force from engine and rotates the powertrain shaft. When clutch is disengaged, the transmission power is cut off, and the shaft rotation stops. With a smooth repetition of the engagement and disengagement processes, a comfortable and energy efficient driving is achieved.

The structure of a generic wet clutch is shown in Fig. 1. Circular disks and clutch plates attached with frictional material are stacked alternately in an outer housing and lubrication oil, that is, Automatic Transmission Fluid (ATF) is supplied between these plates. For an efficient clutch, the following characteristics are required:

1. High friction coefficient for high clutch capacity.

2. Durability for severe conditions.

3. Low drag torque to reduce fuel cost.

The former two requirements are related to the



Fig. 1 Configuration of multi-plate wet clutch 
engagement process of wet clutch. In this direction, a study on frictional material and thermal control with ATF [1] was carried out. This study focused on the development of wet clutch with low drag torque. As the wet clutch pack is filled with viscous oil, the drag torque develops by the sliding shear stress at the disengagement state, and it gives rise to energy loss. This drag torque is linearly proportional to the rotation speed of clutch disk at the low rotation regime and the oil flow is of single-phase. However, if the speed reaches a critical value, air enters into the clutch and the drag torque decreases gradually according to the rotation speed. Although the occurrence of air bubble may also be caused by the reduction of pressure, no bubble was observed at a preliminary experiment with a submerged clutch disk in an oil bath in spite of a very high rotating condition, $3000 \mathrm{~min}^{-1}$. Therefore, the two-phase flow in a wet clutch is caused by the aeration from small gaps of clutch disks in the operating condition and clutch disk configuration of the present study, the drag torque can theoretically be described by

$$
T q=r_{m} \mu \frac{V}{h} A(1-\alpha)
$$

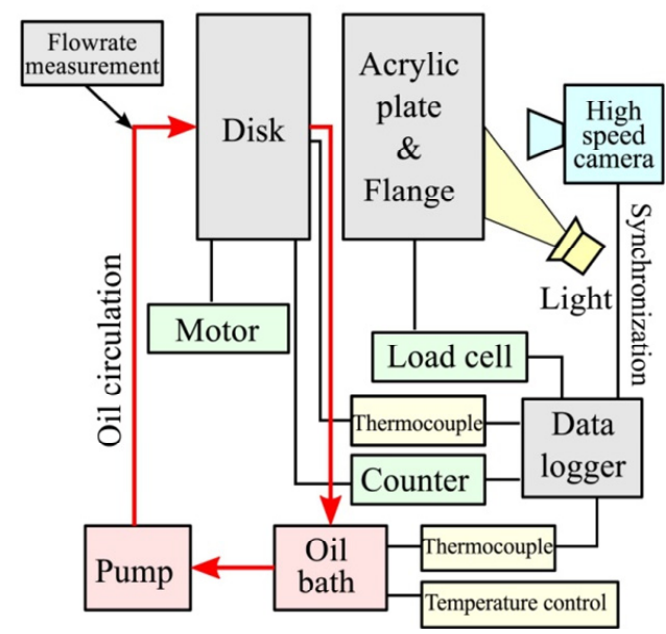

(a) block diagram of system

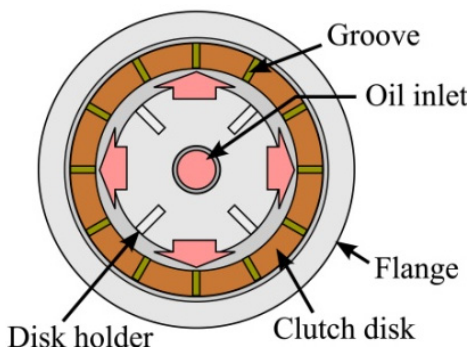

(c) top view of clutch disk
Although most parameters in this equation are known from the operating conditions and clutch geometry, the volume fraction of induced air, $\alpha$, cannot easily be determined. It is also difficult to predict the transition from the single-phase flow regime to the two-phase one. In order to address these issues, analytical estimations for the drag torque curve were proposed [2-5], and three-dimensional hydrodynamic simulations (considering the groove structures in a friction plate) $[6,7]$ were carried out. In spite of these efforts, the general estimation of the drag torque curve was not performed, and the effect of two-phase flow on the drag was not determined. Therefore, in order to shed light on these issues, in this study we investigated the effect of geometrical and operating conditions on the drag torque of wet clutch by means of a combined numerical and experimental study.

\section{Experimental Setup}

In the present study, the single wet clutch system shown in Fig. 2 was used because it is the minimum unit of a multi-plate wet clutch system seen in Fig. 1. Figure 2(a) shows the block diagram of drag torque measurement system and Figs. 2(b) and 2(c) show the

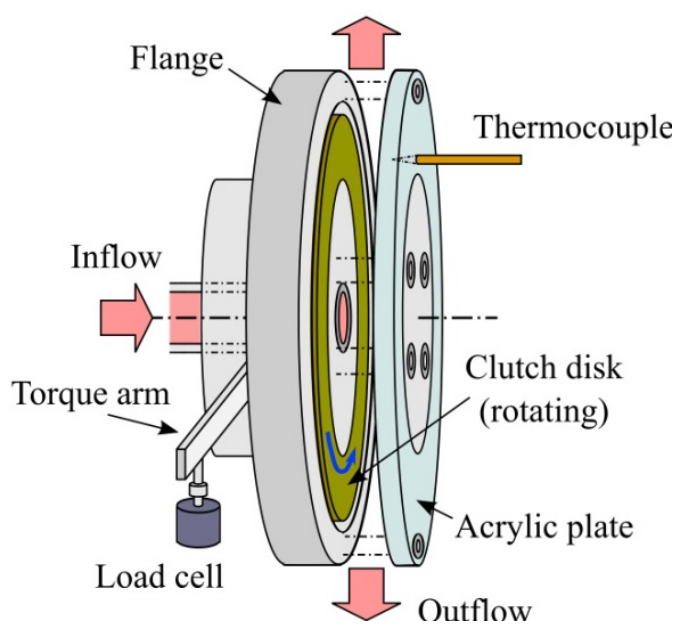

(b) perspective view of clutch disk

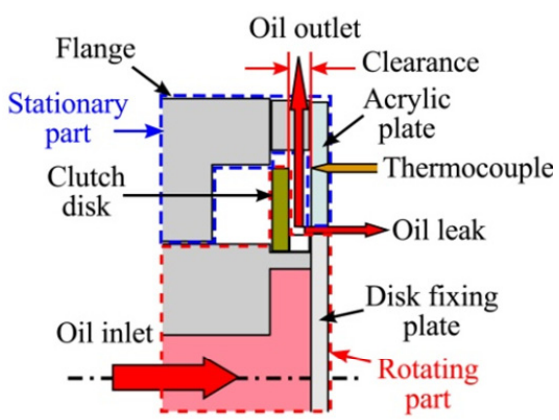

(d) enlarged cross-section view of clutch disk

Fig. 2 Configuration of drag torque measurement experiment 
Table 1 Dimensions of clutch disk

\begin{tabular}{ll}
\hline Property & Value \\
\hline Inner radius & $47.5 \mathrm{~mm}$ \\
Outer radius & $59.5 \mathrm{~mm}$ \\
Number of radial groove & $0,4,12,24$ \\
& None, $9^{\circ}, 3^{\circ}$, \\
Angular width of radial groove & $1.5^{\circ}$ \\
Number of circumferential groove & 0,1 \\
Width of circumferential groove & $1.0 \mathrm{~mm}$ \\
Radius of circumferential groove & $54.0 \mathrm{~mm}$ \\
Groove depth & $0.37 \mathrm{~mm}$ \\
\hline
\end{tabular}

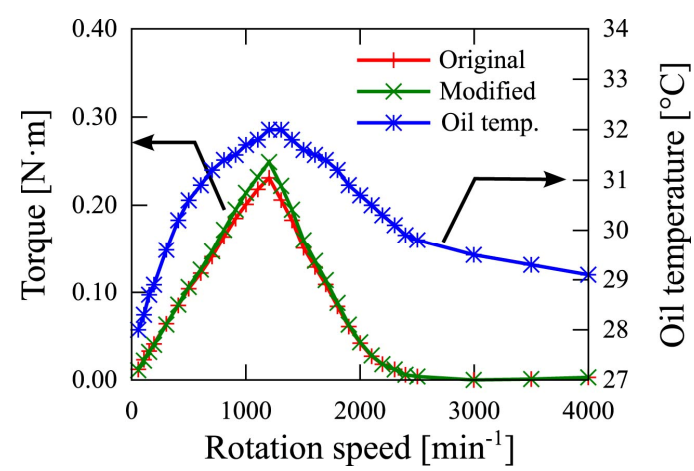

Fig. 3 Dependency of ATF temperature variation on drag torque

perspective and top views, respectively, and Fig. 2(d) shows the enlarged cross-section view of clutch disk. Dimensions of the clutch disk are presented in Table 1. Several segments made of paper frictional material were attached on a metal plate that rotates. The grooves between segments were placed at equal distances from each other, and the angle width of radial grooving was varied according to the number of grooves in order to make the total area of the grooves same in each case. Width of the clearance was fixed by the plate (Fig. 2(d)) though it varies in an actual clutch pack. ATF is supplied from the guide inlet at the axial center and ejected outward in the radial direction as shown in Figs. 2(b) and 2(c). The ejected oil was stored in an oil bath and recycled as shown in Fig. 2(a), however, it was not saturated by air. Drag torque was measured with the load cell through the torque arm (Fig. 2(b)) and recorded in the data logger, and the averaged value for a 10 -second operation at a constant rotation speed was used. The ATF temperature was measured at the target point on the disk (Figs. 2(b) and 2(c)) and the oil bath by thermocouple, and the supplied ATF temperature into the clutch disks was controlled with an ON-OFF controller equipped with the oil bath. To check the effect of viscous heating on the clutch disks, the dependency of temperature fluctuation on drag torque is shown in Fig. 3. The test was conducted with smooth clutch disk (flow rate was $200 \mathrm{ml} / \mathrm{min}$, width of the clearance was $0.20 \mathrm{~mm}$ ). Although the ATF temperature was controlled in the oil bath, the measured
Table 2 Physical properties of ATF

\begin{tabular}{ll}
\hline Property & Value \\
\hline Temperature & $30{ }^{\circ} \mathrm{C}$ \\
Density & $870 \mathrm{~kg} / \mathrm{m}^{3}$ \\
Kinematic viscosity & $41.5 \times 10^{-6} \mathrm{~m}^{2} / \mathrm{s}$ \\
\hline
\end{tabular}

Table 3 Measurement conditions of the single clutch disk test

\begin{tabular}{ll}
\hline Condition & Value \\
\hline Frictional material & F. C. C. product \\
Oil flow rate & $100,200,300 \mathrm{ml} / \mathrm{min}$ \\
Clearance & $0.20,0.25,0.30 \mathrm{~mm}$ \\
Rotation speed & $0-4000 \mathrm{~min}^{-1}$ \\
\hline
\end{tabular}

temperature on the clutch disk changed from $28^{\circ} \mathrm{C}$ to $32^{\circ} \mathrm{C}$ for the targeted value of $30^{\circ} \mathrm{C}$. In Fig. 3, the original drag torque means the value obtained from the load cell and the modified drag torque was normalized with the ratio of the viscosity at $30^{\circ} \mathrm{C}$ to the viscosity at actual measured temperature. The maximum torque difference was $0.018 \mathrm{~N} \cdot \mathrm{m}$ around $1200 \mathrm{~min}^{-1}$ and the relative error was $7.8 \%$. Therefore, it is noted that the effect of viscous heating on drag torque may appear within an acceptable margin of error and the drag torque may be underestimated around the torque peak.

A part of supplied ATF leaks through a small gap between the acrylic plate and the disk fixing plate at low rotation speed. On the other hand, at high rotation speed, ATF becomes insufficient between the plates and air is brought from the small gap. Physical properties of ATF are summarized in Table 2. The solubility of air into general oil is about $10 \%$ under atmospheric pressure and increases with pressure, however, such a cavitating effect was not considered in the present study. All measurement conditions are listed in Table 3. When the dependency of clearance or flow rate was investigated, either condition was fixed. The acrylic plate was applied at the stationary plate without frictional material and the bubble-oil flow was visualized by a high-speed camera.

\section{Numerical simulation}

3.1. Numerical method

Although the flow field in the wet clutch becomes two-phase flow over a certain rotation speed, the numerical simulation of a multi-phase flow however requires huge computational time and also a complicated numerical scheme. Therefore, here we considered a single-phase flow at the low rotation speed regime, and did not take aeration into account.

The ATF used in the present study is regarded as a Newtonian fluid under the operating conditions shown in Table 3. Thus, the governing equations for an incompressible oil flow are given as follows:

Continuity:

$$
\nabla \cdot \mathbf{u}=0
$$


Momentum:

$$
\frac{\partial \mathbf{u}}{\partial t}+\mathbf{u} \cdot \nabla \mathbf{u}=-\frac{1}{\rho} \nabla p+v \nabla^{2} \mathbf{u}
$$

The flow field must be turbulent at high rotation speed, however, the turbulent effect due to the fluctuation of velocity was not considered in Eq. (3) because it is assumed that the mean flow is dominant between the parallel plates for such high shear flow. The above governing equations were discretized by the finite volume method in an unstructured grid system and solved by the SIMPLE algorithm. The computational domain was the clearance between the two parallel plates. As shown in Fig. 4, the divided region with a radial groove was considered to reduce computational cost. Boundary conditions of the velocity and pressure fields are presented in Fig. 4. At the inlet, the velocity was calculated from the flow rate and the condition of pressure was the Neumann type. On the other hand, at the outlet, the value of pressure was assumed to be equal to the ambient air pressure and the velocity gradient normal to the outer boundary was taken zero. The oil leak at the gap of the stationary plate was not taken into account for simplicity. Drag torque was calculated by the integration of the wall shear stress on the rotated clutch disk. Numerical simulations were carried out using the open source software; OpenFOAM.

\subsection{Verification of simulation code}

The calculation of the test case corresponding to the previous numerical study by Aphale et al. [6] was carried out to verify the present simulation code and assess the grid dependency of the solution. The calculation conditions of the test case are summarized in Table 4 and the physical properties of ATF were the same values as the present experiment values shown in Table 2. As

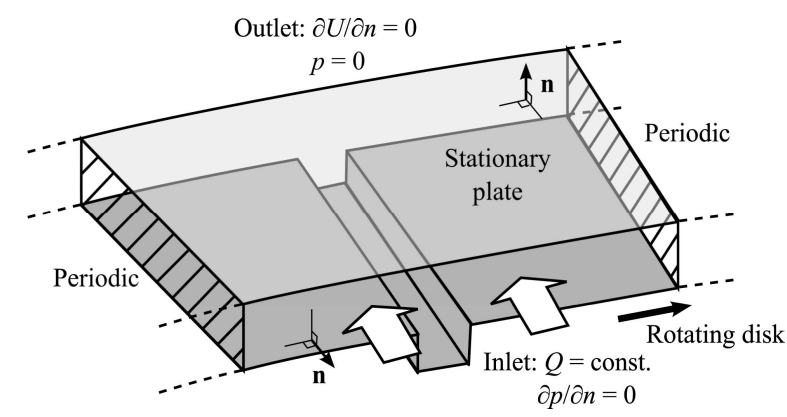

Fig. 4 Numerical model of the drag torque simulation
Aphale et al. [6] used the clutch disk with 40 radial grooves, the computational region of the test case was $1 / 40$ in the circumferential direction, and the plane disks without grooves were used for simplicity. The three computational grid sets as shown in Table 5 were tested, and the resolution of the normal grid was comparable to that of Aphale et al. [6]. The drag torque was calculated by the following integration of wall shear stress:

$$
T q=\int_{A} \tau_{\mathrm{w}} d A=\left.\mu \int_{A} \frac{\partial U}{\partial n}\right|_{\text {wall }} d A
$$

The calculated drag torques at each grid resolutions and the theoretical estimation value by using the equation of Kitabayashi et al. [3] are shown in Table 5. The drag torques slightly decreased as the grid resolutions increased, however, the relative error was less than $1 \%$ and the calculated value was comparable to the theoretical estimation. Therefore, the validity of the present simulation was confirmed and the computational grids corresponding to the normal grids shown in Table 5 were used in the present numerical simulations.

\section{Results and Discussion}

The drag torque curves were obtained from both numerical simulations and experiment. For example, as shown in Fig. 5, the curves of computational results are straight lines and agree with the experimental results at low rotation speeds before the torque peaks. Therefore, the flow field at the low rotation region must be a single-phase flow of liquid oil since the numerical simulation did not consider the contribution of air bubbles.

The effect of clearance on drag torque was investigated and the result was shown in Fig. 5. The flow rate of supplied ATF was fixed at a value of $200 \mathrm{ml} / \mathrm{min}$.

Table 4 Test case for the verification of the simulation code [6]

\begin{tabular}{ll}
\hline Property & Value \\
\hline Inner radius & $72.0 \mathrm{~mm}$ \\
$\begin{array}{l}\text { Outer radius } \\
\text { Division number in the } \\
\text { circumferential }\end{array}$ & $81.3 \mathrm{~mm}$ \\
direction & 40 \\
Oil flow rate & $100 \mathrm{ml} / \mathrm{min}$ \\
Clearance & $0.10 \mathrm{~mm}^{-1}$ \\
Rotation speed & $1000 \mathrm{~min}^{-1}$ \\
\hline
\end{tabular}

Table 5 Grid dependency of calculated torques

\begin{tabular}{lll}
\hline Label & Grid number $\left(N_{r} \times N_{\theta} \times N_{z}\right)$ & Torque $[\mathrm{N} \cdot \mathrm{m}]$ \\
\hline Coarse & $11 \times 29 \times 7$ & 1.3267 \\
Normal & $21 \times 57 \times 13$ & 1.3262 \\
Fine & $41 \times 113 \times 25$ & 1.3251 \\
Kitabayashi et al. (2003) [3] & & 1.3227 \\
\hline
\end{tabular}




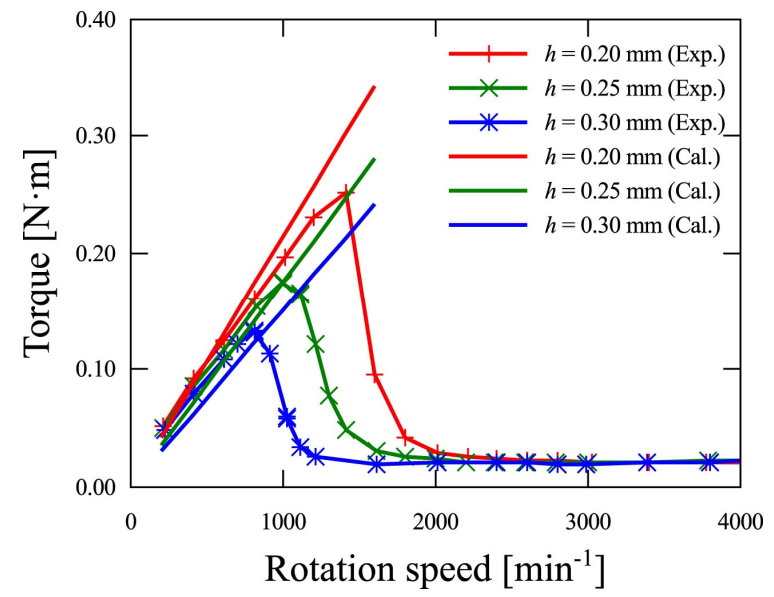

Fig. 5 Effect of clearance width on drag torque

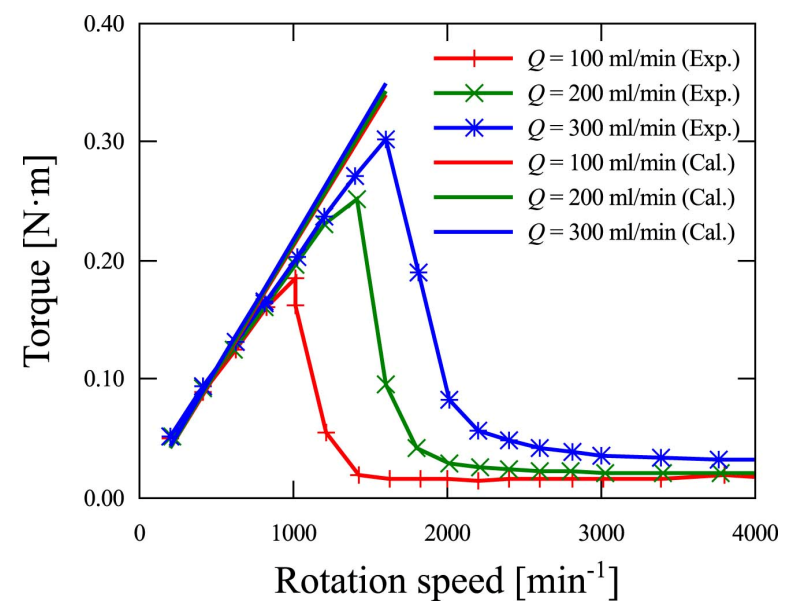

Fig. 6 Effect of flow rate on drag torque

No grooves were made on the clutch disk. The slight difference between numerical and experimental results at lower rotation speed than drag torque peak is seen because the precise keeping of constant clearance in the experiment is difficult at high rotation speed. These small experimental errors in single-phase flow regime are also seen in the following experimental results. The slope of curves at the low rotation region became small as the clearance increased. This is because the velocity gradient on the wall decreases with increasing clearance between two parallel plates for the same condition of sliding velocity. It was found that the drag torque peaks moves to a high rotation speed as the clearance decreases. Because the gap volume between the clutch disks increases as the clearance increases, the large clearance caused oil shortage and aeration at lower rotation speed than the small clearance.

The effect of flow rate on drag torque was shown in Fig. 6. The clearance was fixed as $0.20 \mathrm{~mm}$. As the shear stress in the circumferential direction is dependent only on the angler velocity (without aeration), the slope of curves at the low rotation region were almost identical to each other. The peak of drag torque extended at high flow rates because the amount of supplied oil increased and

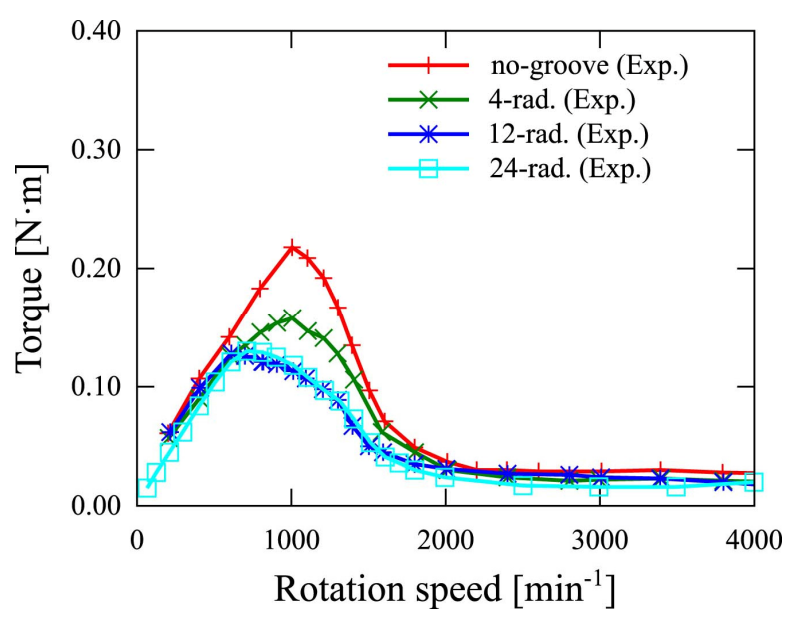

Fig. 7 Effect of radial groove on drag torque
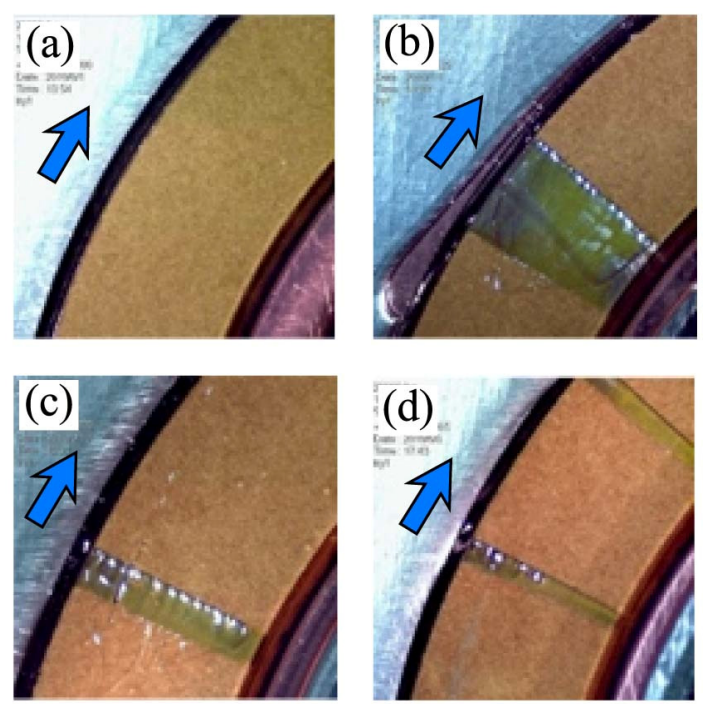

Fig. 8 Visualization of aeration on the clutch disk at $600 \mathrm{~min}^{-1}$ : (a) no grooves, (b) 4 radial grooves, (c) 12 radial grooves, (d) 24 radial grooves

the injection of air from surrounding was delayed.

It is known that the groove formed by frictional material segments plays an important role in controlling oil flow in the wet clutch. The drag torque curves with radial grooves were shown in Fig. 7. The introduction of radial grooving shifted the peak point towards to the low rotation speed region and made the peak value lower. To observe the flow field around a radial groove, the visualized bubble-oil flows are shown in Figs. 8 and 9. At $600 \mathrm{~min}^{-1}$, although the illuminated air bubbles were seen at the edge of groove, the streak of bubble was hardly seen in the main stream. The bubble volume fraction was not strongly dependent on the number of grooves, and the torque values at $600 \mathrm{~min}^{-1}$ were almost in the same order as shown in Fig. 7. From Fig. 9, at $1000 \mathrm{~min}^{-1}$, the bubble volume fractions were significantly large with 12 or 24 radial grooves and the bubbles were also seen on the 

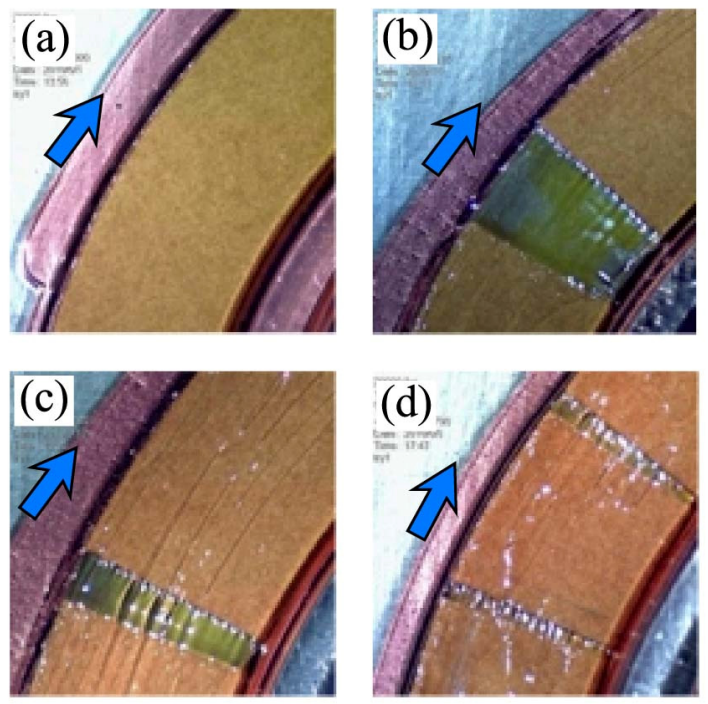

Fig. 9 Visualization of aeration on the clutch disk at $1000 \mathrm{~min}^{-1}$ : (a) no grooves, (b) 4 radial grooves, (c) 12 radial grooves, (d) 24 radial grooves

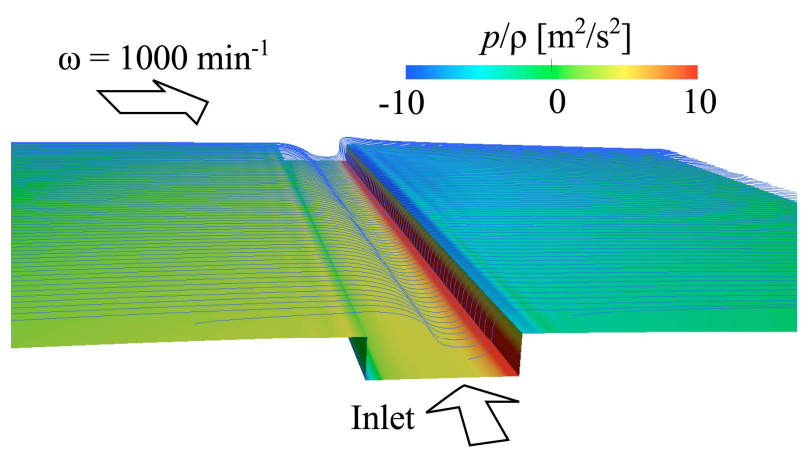

Fig. 10 Pressure and streamlines distributions on the clutch disk surface with 24 radial grooves at $1000 \mathrm{~min}^{-1}$

segments clearly. The pressure distribution on the clutch disk surface and the computed streamlines near the wall were shown in Fig. 10. The strong pressure variation appeared around the edge of radial grooving and the entrained fluid motion was observed. Thus, it appears that this imbalance enhanced the aeration and increased the volume fraction of air $(\alpha)$. As a result, the drag torque was reduced as the theoretical drag torque curve of Eq. (1) indicates. It is noted that the pressure distribution in the present simulation indicates a relative value to the ambient pressure and is not directly related with the solubility of air into the ATF. Although a certain amount of air is solved in the ATF and the solubility depends on pressure and temperature, the precise two-phase simulation including its effect takes many numerical costs and was not carried out in the present study. The ejection of oil was enforced along the radial groove, and the scarce of oil was easily caused compared with the

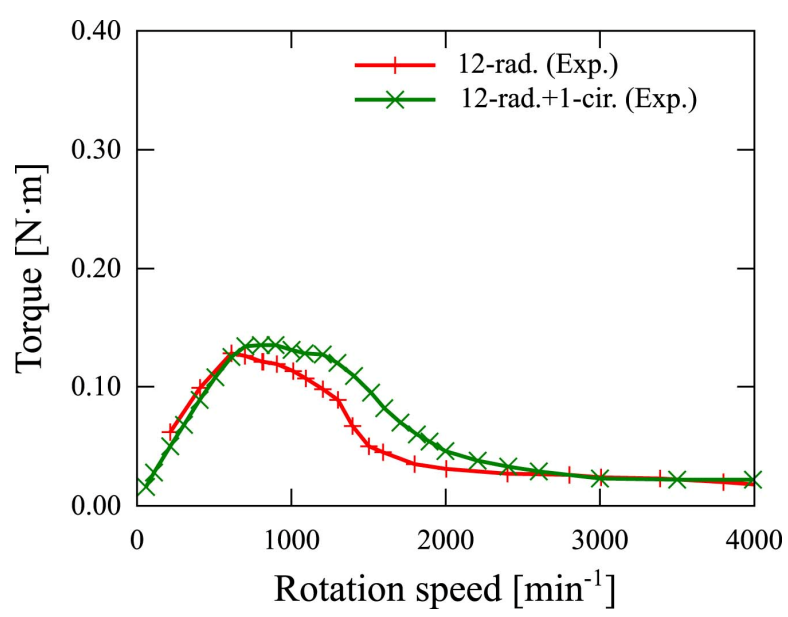

Fig. 11 Effect of circumferential groove on drag torque
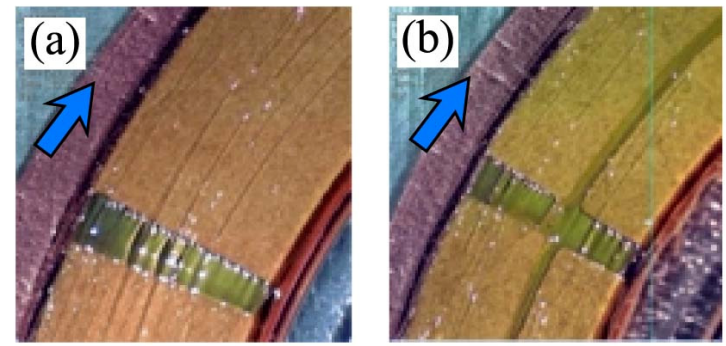

Fig. 12 Reduction of aeration by circumferential groove at $1000 \mathrm{~min}^{-1}$ : (a) 12 radial grooves, (b) 12 radial and one circumferential grooves

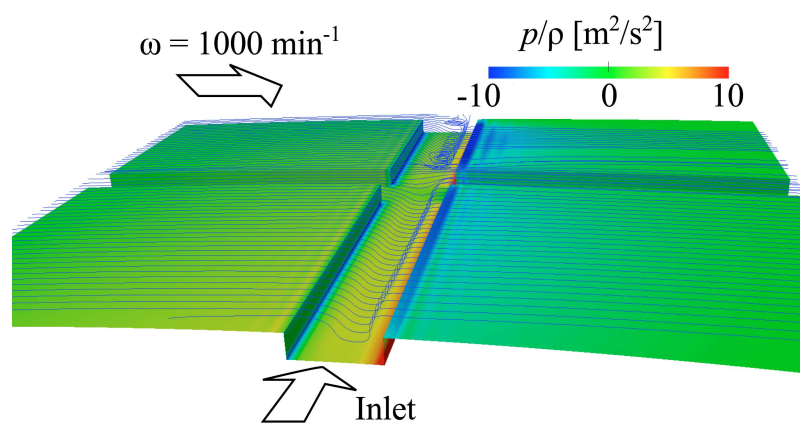

Fig. 13 Pressure and streamline distributions on the clutch disk surface with 24 radial and one circumferential grooves at $1000 \mathrm{~min}^{-1}$

no-groove case.

Finally, the effect of circumferential groove was investigated and the drag torque curves were shown in Fig. 11. The difference of torque was not seen before the peak. However, the introduction of circumferential groove led to an increase in torque at the two-phase flow region. From the visualization of Fig. 12, it was found that the air volume fraction decreased drastically and the bubbles on the clutch disk were related to drag torque. 
The pressure contour on the surface and the computed streamlines distribution (with a circumferential groove) were shown in Fig. 13. The streamlines were concentrated around the circumferential groove that had the oil holding effect and it is the opposite characteristic to that of the radial groove.

\section{Conclusions}

The effect of flow field on drag torque in a wet clutch was examined through a combined numerical and experimental study. Three-dimensional simulations of a single-phase flow, and the experimental measurement of drag torque led to the following conclusions.

- At the low rotation speed of clutch disk, the flow field is of single-phase in the absence of air bubbles, and the drag torque curve is linearly proportional to the rotation speed. Clearance between the parallel plates affects the velocity gradient normal to the wall and changes the slope of torque curve.

- Aeration from the surrounding air appears at a certain rotation speed. Then, the drag torque reaches a peak value. After the peak, the drag torque decreases as the volume fraction of induced bubbles increases. The decrease of flow rate at the inlet, and the introduction of radial grooving on the disk surface are effective to move the peak position to lower rotation range and reduce its peak value.

In the advanced study on drag torque in a wet clutch, the relation between the two-phase state of ATF and the drag torque will be discussed more qualitatively and an optimum wet clutch required by the operating conditions will be developed through a combined numerical and experimental approach.

\section{Acknowledgement}

The authors would greatly thank Prof. Sadik Dost at University of Victoria for his critical reading of the manuscript.

\section{Nomenclature}

$\begin{array}{ll}A & \text { Surface area of disk } \\ h & \text { Clearance } \\ N_{r} & \text { Grid number in the radial direction } \\ N_{z} & \text { Grid number in the axial direction } \\ N_{\theta} & \text { Grid number in the circumferential direction }\end{array}$

$\begin{array}{ll}\mathbf{n} & \text { Normal direction to boundary } \\ p & \text { Pressure } \\ Q & \text { Flow rate } \\ r & \text { Radial position } \\ r_{\mathrm{m}} & \text { Mean radius of disk } \\ T q & \text { Drag torque } \\ U & \text { Velocity normal to boundary } \\ \mathbf{u} & \text { Velocity vector } \\ V & \text { Disk velocity } \\ \alpha & \text { Volume fraction of induced air } \\ \mu & \text { Viscosity } \\ v & \text { Kinetic viscosity } \\ \tau_{\mathrm{w}} & \text { Wall shear stress } \\ \rho & \text { Density } \\ \omega & \text { Rotation speed }\end{array}$

\section{References}

[1] Miyagawa, M., Ogawa, M., Okano, Y., Hara, H., Sasaki, S. and Okui, K., "Numerical Simulation of Temperature and Torque Curve of Multidisk Wet Clutch with Radial and Circumferential Grooves," Tribology Online, 4, 1, 2009, 17-21.

[2] Kato, Y., Murasugi, T., Hirano, H. and Shibayama, T., "Fuel Economy Improvement Through Tribological Analysis of the Wet Clutches and Brakes of an Automobile Transmission," SAE Paper, No. 938179, 1993.

[3] Kitabayashi, H., Li, C. and Hiraki, H., "Analysis of the Various Factors Affecting Drag Torque in Multi-Plate Wet Clutches," SAE Paper, No. 2003-01-1973, 2003.

[4] Yuan, Y. Liu, E. A., Hill, J. and Zou, Q., "An Improved Hydrodynamic Model for Open Wet Transmission Clutches," Journal of Fluids Engineering, 129, 2007, 333-337.

[5] Hu, J., Peng, Z. and Yuan, S., "Drag Torque Prediction Model for the Wet Clutches," Chin. J. Mech. Eng., 22, 2, 2009, available online at http://www.cjmenet.com.

[6] Aphale, C. R., Cho, J., Schultz, W. W., Ceccio, S. L., Yoshioka, T. and Hiraki, H., "Modeling and Parametric Study of Torque in Open Clutch Plates," Journal of Tribology, 128, 2006, 422-430.

[7] Yuan, Y., Attibele, P. and Dong, Y., "CFD Simulation of the Flows within Disengaged Wet Clutches of an Automatic Transmission," SAE Paper, No. 2003-01-0320, 2003. 